\title{
Effect of Vitamin A and Methylprednisolone on Canine Prostate in Organ Culture 1
}

THEODORE V. FISCHER, RAYMOND H. KAHN, WILLIAM E. BURKEL, DAVID W. VINTER AND KARL R. HERWIG

Departments of Anatomy and Surgery, The University of Michigan Medical School, Ann Arbor, Michigan 48109

\begin{abstract}
Organ explants from the canine prostate with and without methylprednisolone pretreatment were cultivated for ten days in Trowell's T-8 medium or medium supplemented with testosterone and/or vitamin A. Upon termination of the experiments, explants were fixed and examined histologically. All glands from the most central section of each explant were graded according to epithelial type, and from these grades the proportion of acini with maintained columnar cells was calculated for each explant. Stromal cellular maintenance was also estimated. While a small proportion of explants from the vitamin-treated and methylprednisolone-pretreated groups showed epithelial maintenance, a combination of these treatments significantly increased such maintenance. Stromal maintenance was enhanced with methylprednisolone pretreatment but not by vitamin A. These results are in accord with the hypothesis that methylprednisolone acts to stabilize the lysosomal membrane, thus protecting tissue against the effects of ischemic shock. In protected explants vitamin $\mathrm{A}$ is able to maintain a columnar glandular epithelium. In a subsequent experiment a series of linoleic acid dosages was tested in the presence or absence of vitamin $A$. In neither case was this fatty acid of value in improving epithelial or stromal maintenance.
\end{abstract}

While testosterone treatment has been shown to support rodent prostatic alveoli in organ culture (Lasnitzki, '55a, '69), no response to this hormone occurs with explants of human (McMahon and Thomas, '73) or canine (Fischer et al., '76) prostate. Other factors must act in concert with testosterone in these species to preserve the histologic architecture evident in the intact prostate.

Vitamin A has been shown to be necessary for the production of mucous metaplastic changes in epidermal tissues (DeLuca et al., '72; Fell and Mellanby, '53). Furthermore this vitamin prevents epithelial hyperplasia of the rodent prostate in organ culture maintained in defined media (Lasnitzki, '62) and counteracts the squamous metaplasia normally elicited by the carcinogen, $20 \alpha$ - methylcholanthrene (Lasnitzki, '55b). In the absence of testosterone, the addition of vitamin $A$ to the media alleviated the regression of the rodent prostatic epithelium which normally follows explantation (Lasnitzki, '62; Lasnitzki and Goodman, '74). An object of this study was to determine whether vitamin $A$ is able to enhance an effect of testosterone on explants of canine prostate.

Glucocorticoids have also been found to reduce epithelial regression (Lasnitzki, '65) and promote the formation of secretory granules (Ichihara et al., '73) in the rodent prostate. Moreover, methylprednisolone (MP) has been used in perfusion to protect cadaveric kidneys against ischemic injury, presumably by stabilizing the lysosomal membrane (Miller and Alexander, '73). In view of the postulated protective role of glucocorticoids in cellular shock, and the necessary trauma and ischemia produced in cutting explants, the effect of pretreatment of donor dogs with MP was studied as well.

The essential fatty acids (EFA) have never been included in organ culture media, despite their importance in cellular metabolism. Linoleic acid is defined as an EFA because in

\footnotetext{
Accepted February 14, '78.

'This investigation was supported by the National Cancer Institute Contract Nos. N01.CP-33331 and N01-CP-55720.
} 
TABLE 1

Experimental plan

\begin{tabular}{|c|c|c|c|c|c|c|}
\hline \multirow{3}{*}{$\begin{array}{c}\text { MP } \\
\text { Pretreatment }\end{array}$} & \multicolumn{6}{|c|}{ In vitro treatment groups ${ }^{1}$} \\
\hline & \multirow[b]{2}{*}{$\begin{array}{c}\text { Testosterone } \\
(\mu M)\end{array}$} & \multicolumn{5}{|c|}{ Vitamin A levels $(\mathrm{IU} / \mathrm{ml})^{2}$} \\
\hline & & $\begin{array}{l}\text { (a) } \\
0\end{array}$ & $\begin{array}{l}\text { (b) } \\
25\end{array}$ & $\begin{array}{c}\text { (c) } \\
0\end{array}$ & $\begin{array}{l}\text { (d) } \\
25\end{array}$ & $\begin{array}{l}(e) \\
50\end{array}$ \\
\hline None & $\begin{array}{l}0 \\
5\end{array}$ & $\begin{array}{l}12 / 15(7) \\
13 / 14(7)\end{array}$ & $\begin{array}{l}16 / 16(7) \\
14 / 16(7)\end{array}$ & $\begin{array}{l}13 / 13(6) \\
14 / 14(6)\end{array}$ & $\begin{array}{l}14 / 14(6) \\
14 / 14(6)\end{array}$ & $\begin{array}{l}13 / 14(6) \\
10 / 13(6)\end{array}$ \\
\hline $\begin{array}{l}30 \mathrm{mg} / \mathrm{kg} \\
2 \text { hours prior } \\
\text { to surgery }\end{array}$ & $\begin{array}{l}0 \\
5\end{array}$ & $\begin{array}{l}22 / 22(16) \\
19 / 19(16)\end{array}$ & $\begin{array}{l}15 / 20(14) \\
17 / 18(14)\end{array}$ & $\begin{array}{r}10 / 11(7) \\
7 / 11(6)\end{array}$ & $\begin{array}{r}10 / 11(7) \\
9 / 11(8)\end{array}$ & $\begin{array}{l}7 / 10(6) \\
9 / 13(6)\end{array}$ \\
\hline
\end{tabular}

' Each block contains the number of viable explants/total explants (and dogs) cultivated with that treatment.

${ }^{2}$ In columns c-e, explants were exposed to $100 \mathrm{IU} / \mathrm{ml}$ for one hour prior to cultivation.

its dietary absence changes in lipid metabolism and in mitochondrial structures and enzymes occur (Kengery and Kellum, '65). In EFA-deficient rats, oxidative phosphorylation is uncoupled in liver mitochondria (Klein and Johnson, '54) and they swell more rapidly in vitro in the isolation medium than do mitochondria from control rats (Johnson, '63). Similar findings have been made in mice (Wilson and Leduc, '63). Some of the functions of EFA's can be explained by their use as precursors of prostaglandins (Pong and Levine, '77). While probably not their sole function, it seems likely that this conversion may be an important expression of EFA activity.

Since the EFA's seem so important for the cell, their inclusion in a basic organ culture medium needs to be investigated. However, since they are very susceptible to peroxidation to intracellular pigments, the antioxidant, vitamin $\mathrm{E}$, would also need to be included in any study of EFA effects.

\section{MATERIALS AND METHODS}

Prostate glands from 23 purebred beagles at one year of age were removed aseptically and prepared for organ culture as previously described (Fischer et al., '76). Sixteen donors were treated two hours preoperatively with $30 \mathrm{mg} / \mathrm{kg}$ body weight methylprednisolone sodium hemisuccinate (Solu-Medrol, Upjohn). Explants were cultivated for a minimum of nine days in Trowell's T-8 medium while being exposed to a continuous gas flow of $50 \%$ oxygen, $5 \%$ carbon dioxide and the remainder nitrogen. Retinol $(3,000 \mathrm{IU} / \mathrm{mg})$ was dissolved in ethanol $(20 \mathrm{IU} / \mu \mathrm{l})$ and varying amounts of this stock solution were added to the culture media (table 1). Some explants were exposed to $100 \mathrm{IU}$ vitamin $\mathrm{A} / \mathrm{ml}$ for one hour prior to cultivation. Subsequently, explants were cultivated in T.8 medium alone or supplemented with 25 or 50 IU of the vitamin $/ \mathrm{ml}$. Testosterone, dissolved in a minimum of ethanol, was added to the media in some experimental groups at a final concentration of 5 $\mu \mathrm{M}$ (table 1). After termination of the cultures all explants were evaluated and compared histologically to the preculture controls.

In a subsequent experiment, the effect of varying levels of the essential fatty acid, linoleic acid, was tested in media containing or lacking vitamin $\mathrm{E}$ ( $\alpha$-tocopherol $\mathrm{Na}$ succinate, $44 \mu \mathrm{M})$. All explants in this experiment were derived surgically from the prostates of six dogs treated two hours preoperatively with $30 \mathrm{mg} \mathrm{MP} / \mathrm{kg}$. Linoleic acid was dissolved in ethanol and added to the culture media at final concentrations of $0.003,0.03,0.3,3$ and $30 \mu \mathrm{M}$. A linoleic acid-free control group completed the series. Vitamin A was added to the media in half of the culture dishes of each group at a concentration of $25 \mathrm{IU} / \mathrm{ml}$. Other culture and evaluation conditions were as described above.

All tissues were fixed in alcohol-formalin. acetic acid (3:1:1), and paraffin sections were stained with hematoxylin and eosin for study. Explants were evaluated by a double-blind procedure, and in the most central section of each explant all glandular acini were classified as having one of the following epithelial cellular types: simple or stratified and either columnar, cuboidal or squamous. If necrotic or degenerative acini were present, they were recorded as such. The proportion of all acini having epithelia of each of the categories was calculated for each explant. If the explant consisted only of necrotic or degenerative acini, it was considered non-viable. 
Because the most uniform morphological feature of normal canine prostate is its tall columnar glandular and duct epithelium (fig. 4), this characteristic was chosen as the chief criterion of maintenance in culture. The mean proportion of columnar glandular acini per explant was calculated for each experimental group, and these means were compared statistically by analysis of variance. In many cases the Student $t$-test was also performed to compare selected pairs of groups. Other observations of all explants included an evaluation of the stromal status. In the same section wherein the epithelium was rated, stromal cells (fibroblasts, smooth muscle and vascular structures) were subjectively classified as: (1) good (no sign of degeneration), (2) poor (some pyknotic cells to completely necrotic stroma). The proportion of explants in each group with "good" stroma was evaluated statistically by the Chi-square test. In addition, Chi-square tests were performed to evaluate the proportion of viable explants in each experimental group.

\section{RESULTS}

Most canine prostatic explants exhibit considerable histologic variability after nine days in culture. In a single explant, some glandular acini may lack an epithelial lining, others may be surfaced with a simple cuboidal epithelium while some may demonstrate the simple columnar epithelium characteristic of the prostate in vivo (fig. 5).

Overall explant viability was quite good in these experiments. A small but significant ( $P$ $<0.01$ ) decrease in the proportion of viable explants occurred in the MP-vitamin A groups ( $73 \%$ viable) when compared to that of groups with either treatment alone $(90 \%)$. However, none of these treatment groups varied significantly from the untreated control group $(80 \%$ viable).

Since no statistically significant differences occurred between explants pretreated with vitamin A for one hour prior to culture and those not pretreated, all explants of the same vitamin A dose level were combined in the following analysis. At the doses used, testosterone-treated explants could not be discriminated from those not treated, and are considered together.

Figure 1 illustrates the effect of MP pretreatment and vitamin $\mathrm{A}$ on the degree of columnar epithelial cell maintenance. Analyses of variance indicate that vitamin A treat-

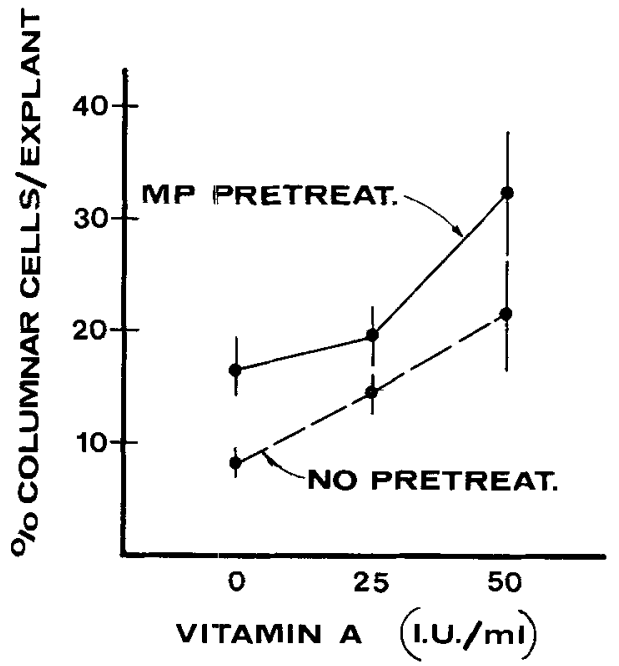

Fig. 1 The effect of MP pretreatment of prostate donor dogs and of vitamin A upon maintenance of columnar glandular epithelium in culture.

ment significantly increases glandular maintenance in groups pretreated $(P<0.01)$ or not pretreated $(\mathrm{P}<0.05)$ with MP. It is obvious also from figure 1 that MP pretreatment has a favorable influence upon explant maintenance, but Student $t$-tests indicate that it is significant only at the vitamin A zero dose level $(P<0.01)$. However, MP does show an overall significant effect $(P<0.01)$ in enhancing epithelial maintenance.

A definite synergistic effect exists between MP-pretreatment and the vitamin, since $23 \%$ of all the glandular acini from the 84 viable explants of these groups was well maintained as columnar epithelium (figs. 1, 6), significantly greater $\left(\mathrm{P}^{\prime}<0.001\right)$ than the $8 \%$ columnar epithelium in the 25 controls (figs. 1,7 ). This contrasts with the $14 \%$ columnar epithelial maintenance which occurred in the 108 explants of the vitamin A groups and the 13\% in the 41 explants of the MP group without vitamin $A$, neither of which differed significantly from the controls.

Although explants from the MP and vitamin groups were similar in the degree of columnar epithelial maintenance, they differed significantly in their stromal status as evaluated by Chi-square analysis of the proportion of explants with stroma rated "good" (no evidence of degeneration) (fig. 2). Whereas the overall effect of: MP was to maintain the viability of stromal cells $(\mathrm{p}<0.001)$ as compared to explants from control animals, the 


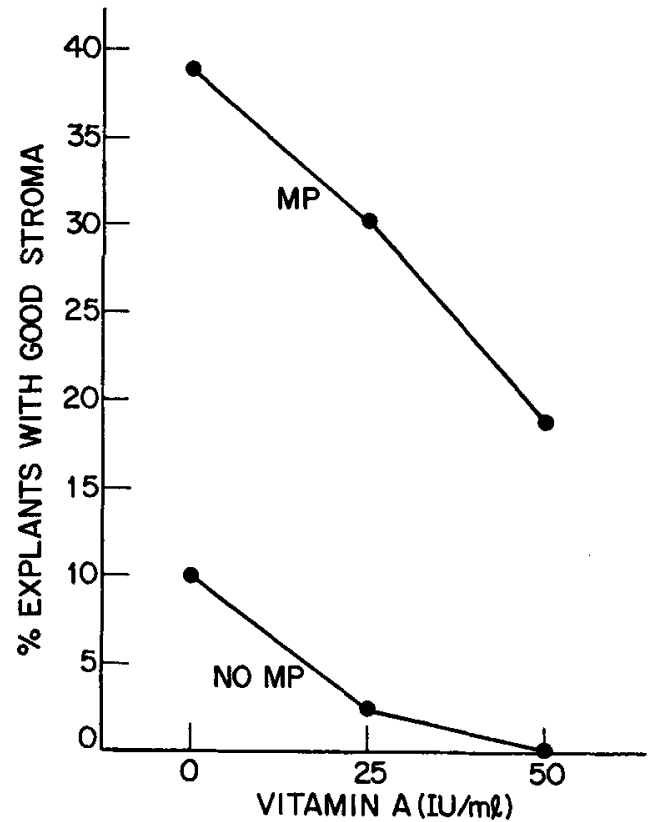

Fig. 2 The effect of MP pretreatment and vitamin A upon the proportion of explants showing no evidence of stromal degeneration.

overall effect of vitamin A treatment was to reduce stromal maintenance $(p<0.01)$ from the level of non-treated control explants. The beneficial effect of MP pretreatment was sig- nificant for all dose levels of vitamin A: 0 (p $<0.01), 25 \mathrm{IU} / \mathrm{ml}(\mathrm{p}<0.001)$ and $50 \mathrm{IU} / \mathrm{ml}$ ( $p<0.05$ ). On the other hand, the detrimental effect of vitamin A upon stromal cell viability occurred in both the MP and non-MP explant groups $(\mathrm{p}<0.05)$. The vitamin's destructive action upon stroma was so great that of the nineteen explants treated with the high dose $(50 \mathrm{IU} / \mathrm{ml})$ of vitamin $\mathrm{A}$, none from the nonMP animals contained normal stroma. Even in MP-pretreated explants, the high dose of vitamin A cut the proportion of explants with maintained stroma in half, compared to vitamin-free controls. It is of interest that many explants in which the stroma was entirely degenerate still maintained their glandular epithelium (fig. 8).

The results of the linoleic acid experiment are summarized in figure 3 . Although linoleic acid is considered an essential fatty acid (but nevertheless is not included in most organ culture media), no effect at all was detected over a wide range of additive dose levels. An important effect of vitamin $E$ is to protect linoleic acid against oxidation. For this reason, vitamin E $(25 \mu \mathrm{g} \mathrm{d}$ - $\alpha$-tocopherol $/ \mathrm{ml})$ was included in half of the linoleic acid pretreatment dishes, yet no difference in maintenance was detected in the presence or absence of vitamin E. However, when vitamin A was included in the medium $(25 \mathrm{IU} / \mathrm{ml})$, there was

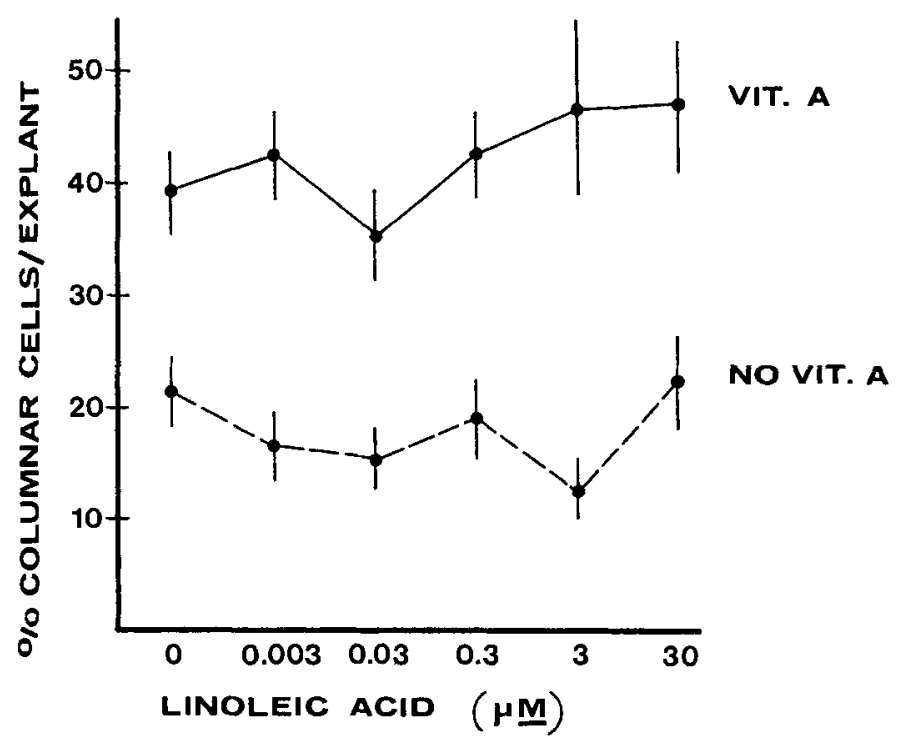

Fig. 3 The effect of varying dosages of linoleic acid on explants treated or not treated with 25 IU vitamin A/ml during culture. All explants are from dogs pretreated with MP. 
uniform improvement in maintenance at every dose level of linoleic acid (fig. 3).

\section{DISCUSSION}

The structural diversity of organ culture explants is well known by those experienced in this field. Within a single explant, a range of structural variation is frequently evident. Nevertheless, a description of the explant is essential if any systematic evaluation of treatment is to be made. In the past, much of this description has been largely qualitative, allowing observer biases to intrude in to the interpretation of the explant's condition. Statements often appear in the literature that a certain hormone or other substance improves explant maintenance but no objective, quantitative criteria are advanced to justify that assessment (Sweeney and Hardy, '76; Lasnitz$\mathrm{ki}$, '72). The evaluation procedures described here for the prostate, while not totally quantitative, do hold observer bias to a minimum and allow a more objective assessment of the degree of tissue maintenance. These procedures include: (1) a double-blind evaluation of all explants; (2) a specific list of observable variables with defined limits for each; (3) the use of a multiple channel counter for tabulation of epithelial states, permitting calculation of the proportion of glands in each category; and (4) the use of a computer system, TAXIR (Brill, '75), to store data by category and organize it for easy statistical analysis.

Vitamin A has long been recognized for its effects on both epithelia and connective tissue in vivo as well as in vitro. The most obvious actions include the regulation of epithelial differentiation (DeLuca et al., '72; Fell and Mellanby, '53; DeLuca and Wolf, '72) and the restoration of wound healing capability inhibited by glucocorticoids (Hunt et al., '69; Ehrlich et al., '73). At the cellular level, vitamin A has been considered a labilizer of lysosomal membranes and consequently responsible for release of acid phosphatase from the cell (Rundell et al., '74; Bassett and Packer, '65). This labilization occurs both in vitro (Daniel et al., '66) and in vivo (Thomas et al., '60), and may be a property of this vitamin with all biomembranes (Lucy, '69).

The classical study of Fell and Mellanby ('52) demonstrated resorption of bone and cartilage upon inclusion of vitamin $A$ in culture. The results of our experiments also show that vitamin $A$ is detrimental to connective tissue, with or without MP (fig. 2). The tendency toward stromal. destruction seen in our explants is reminiscent of vitamin $A$ action on limb bone rudiments in culture (Fell and Thomas, '60) and may be explained as the release of lysosomal proteolytic enzymes into the intercellular matrix. The discontinuities of the basal lamina and the extremely large amounts of stromal cellular debris seen in electron micrographs of such explants suggest a pattern of destruction attributable to lysosomal enzyme liberation (Klann et al., '77).

On the other hand, other studies have suggested that vitamin $\mathrm{A}$ is a lysosomal stabilizer at an optimal concentration, while above and below that optimum the membranes become unstable and release lysosomal contents (Sud. hakaran and Kurup, '74). Because of the extreme instability of this vitamin, regulation of its effective concentration in organ culture becomes difficult. Our dosages were based on those previously used successfully in cultivation of rodent prostate (Lasnitzki, '62) and skin (Barnett and Szabo, '73). The detrimental stromal effects of vitamin $A$ in our experiments may be due to a pharmacologic overdose, and a much lower dosage may prove to be optimal for the canine prostate.

Among the many well-known effects of glucocorticoids is their ability to stabilize the lysosomal membrane (Weissmann, 72). It is because of this property that the glucocorticoid, MP, has become widely used to protect organs for transplantation from the damaging cellular effects of ischemic shock (Miller and Alexander, '73; Sterling et al., '73). Although it is not widely appreciated, organ explants prepared for culture are subjected to the same ischemic conditions as are whole organs, and are likewise extremely susceptible to cellular shock. It was to protect prostatic explants from the effects of shock that MP was given to donor dogs prior to prostatectomy.

While a minimal epithelial effect could be attributed to MP alone (fig. 1), its action as a lysosomal stabilizer in shock alleviation (Sterling et al., '73) was consistent with our experimental findings showing a marked stromal cellular improvement in explants from MP pretreated dogs (fig. 2). It is important to note that we did not use MP as a culture medium additive, since studies of inflammation and wound healing indicate that glucocorticoids actually inhibit collagen synthesis and connective tissue repair (Ehrlich et al., '73; Stephens et al., '73). Experiments in which this was done did show suppression of stromal 
growth (Lasnitzki, '62) and, in fact, our preliminary results showed MP to be detrimental when included in the medium.

The most striking finding of this study, however, was the interaction of MP pretreatment and vitamin A medium supplementation. Whereas both the vitamin and MP treatments had minor effects upon epithelial maintenance when given separately, a marked improvement in maintenance occurred when they were combined (fig. 1). Nearly one third of the glandular epithelium was maintained in a histotypical manner quite similar to the pre-culture control tissue. Whereas the rodent prostate was able to respond to vitamin $\mathrm{A}$ following the trauma of surgical removal and division into explants (Lasnitzki, '62), the same could not be said of the canine prostate. Explants from untreated dogs may have been in such a condition of physiological shock that they were able to respond only weakly to vitamin A during cultivation. On the other hand, prostatic explants taken from dogs pretreated with MP have had the benefit of its protective action on lysosomal membranes, allowing an increased definite response to the added vitamin $A$.

A total lack of effect of the essential fatty acid, linoleic acid, was observed. Since no effect occurred over a 10,000-fold dosage range, and since no difference existed between groups treated with the anti-oxidant, vitamin $\mathrm{E}$, and those not so treated, it must be concluded that either a sufficient endogenous supply of linoleic acid was present or that it is not really "essential" to canine prostate in vitro.

Statistically significant differences in response have always occurred between explants from different dogs $(p<0.01)$. Because the linoleic acid experiment was performed on explants from six dogs not used in the first vitamin A/MP experiment, it is not surprising that the general maintenance level seemingly differs between these experiments (figs. 1, 3). Nevertheless, within each experiment, vitamin A treatment resulted in an increase in the level of epithelial maintenance.

Testosterone added to the medium did not sustain an epithelium comparable to that of the intact canine prostate, unlike its effect on rodent explants (Lasnitzki, '55). This agrees with a recently published study (Sinowatz and Pierrepoint, '77) as well as our own earlier observations (Fischer et al., '76), even though the reasons for this lack of effect remain unclear. It is well known that circulating testosterone in vivo is taken up by prostatic epithelial cells and metabolized to yield more active androgens (Bruchovsky and Wilson, '68a). In rodents, this active form is $5 \alpha$-dihydrotestosterone (DHT) (Bruchovsky and Wilson, '68b). Although DHT is present in the dog prostate in large amounts (Morfin et al., '70), current evidence points to $5 \alpha$-androstan$3 \alpha, 17 \alpha$-diol as the active androgen in this species (Evans and Pierrepoint, '75, '76). Even though testosterone was included in our media, it may not have been adequately taken up or metabolized to a functional form by the target cells. It is therefore of great interest that Sinowatz and Pierrepoint ('77) have shown that only $5 \alpha$-androstan- $3 \alpha, 17 \alpha$-diol has a definite effect in maintaining a secretory epithelium. Experiments in our laboratories are now in progress to confirm this report.

\section{LITERATURE CITED}

Barnett, M. L., and G. Szabo 1973 Effect of vitamin A on epithelial morphogenesis in vitro. Fine structural changes in explants of adult mammalian skin. Exptl. Cell Res., 76: 118.126.

Bassett, B. E., and L. Packer 1965 Response of isolated lysosomes to vitamin A. J. Cell Biol., 27: 448-450.

Brill, R. C. 1975 The Taxir Primer. Center for Human Growth and Development, The University of Michigan, Ann Arbor, Michigan.

Bruchovsky, N., and J. D. Wilson 1968a The conversion of testosterone to $5 \alpha$-androstan-17 $\beta$-ol-3-one by rat prostate in vivo and in vitro. J. Biol. Chem., 243: 2012-2021.

1968b The intranuclear binding of testosterone and $5 \alpha$-androstan-17 $\beta$-ol-3-one by rat prostate. J. Biol. Chem., 243: 5953-5960.

Daniel, M. R., J. T. Dingle, A. M. Glavert and J. A. Lucy 1966 The action of excess vitamin A alcohol on the fine structure of rat dermal fibroblasts. J. Cell Biol., 30: 465-475.

DeLuca, L., and G. Wolf 1972 Maintenance of epithelial cell differentiation: the mode of action of vitamin $A$. Cancer, 30: 1326-1331.

Ehrlich, H. P., H. Tarver and T. K. Hunt 1973 Effects of vitamin $A$ and glucocorticoid upon inflammation and collagen synthesis. Ann. Surg., 177: 222-227.

Evans, C. R., and C. G. Pierrepoint 1975 Demonstration of a specific cytosol receptor in normal and hyperplastic ca-

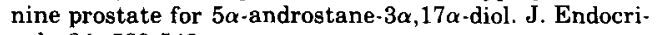
nol., 64: 539-548.

1976 Studies on the uptake and binding of $5 \alpha$ androstane- $3 \alpha, 17 \alpha$-diol by canine prostatic nuclei. J. Endocrinol., 70: 31-37.

Fell, H. G., and E. Mellanby 1952 The effect of hypervitaminosis $A$ on embryonic limb-bones cultivated in vitro. J. Physiol., 116: 320-349.

1953 Metaplasia produced in cultures of chick ectoderm by high vitamin A. J. Physiol., 119: 470-488.

Fell, H. B., and L. Thomas 1960 Comparison of the effects of papain and vitamin $A$ on cartilage. II. The effects on organ cultures of canine prostate. J. Exp. Med., 111 . 719-744.

Fischer, T. V., W. E. Burkel, R. H. Kahn and K. R. Herwig 
1976 Effect of testosterone on long term organ culture of canine prostate. In Vitro, 12: 382-392.

Hunt, T. L., H. P. Ehrlich, J. A. Garcia, and J. E. Dunphy 1969 Effect of vitamin A on reversing the inhibitory effect of cortisone on healing of open wounds in animals and man. Ann. Surg., 170: 633-641.

Ichihara, I., R. S. Santti, and L. J. Pelliniemi 1973 Effects of testosterone, hydrocortisone and insulin on fine structure of epithelium of rat ventral prostate in organ-culture. Z. Zell. Mikr. Anat., 143: 425-438.

Johnson, R. M. 1963 Swelling studies on liver mitochondria from essential fatty acid deficient rats. Exp. Cell Res., 32: 118-129.

Kengery, F. A., and R. E. Kellum 1965 Essential fatty acid deficiency. Arch. Derm., 91: 272-279.

Klann, R. C., W. E. Burkel, T. V. Fischer, K. R. Herwig and R. H. Kahn 1977 Ultrastructural effects of hormones and vitamin A on the canine prostate in vitro. Anat. Rec., 187: 626.

Klein, P. D., and R. M. Johnson 1954 Phosphorus metabolism in unsaturated fatty acid-deficient rats. J. Biol. Chem., 211: 103-110.

Lasnitzki, I. 1955a The effect of testosterone proprio. nate on organ culture of the mouse prostate. J. Endocrinol., 12: 236-240.

- 1955b The influence of a hypervitaminosis $A$ on the effect of 20 -methylcholanthrene on mouse prostate glands grown in vitro. Brit. J. Cancer, 9; 434-441.

1962 Hypervitaminosis $A$ in mouse prostate gland cultured in chemically defined medium. Exptl. Cell Res., 28: 40-51.

1965 Action and interaction of hormones and 3 methylcholanthrene on the ventral prostate of the rat in vitro. II. Hydrocortisone and methylcholanthrene. J. Natl. Cancer Inst., 35: 1001-1014.

1969 The action of testosterone and its metabolites on the rat prostate gland grown in organ culture. In: D. C. Workshop Conference-Advances in the Study of the Prostate. D. C. Williams, M. H. Briggs and M. Staniford, eds. Heineman Medical Books, London, 1969, pp. 65-72.

1972 The effect of prolactin on rat prostate glands grown in organ culture. In: Proc. of the Fourth Tenovus Workshop-Prolactin and Carcinogenesis. A. R. Boyns and K. Griffiths, eds. Alpha Omega Publishing, Cardiff, pp. 200-206.

Lasnitzki, I., and D. S. Goodman 1974 Inhibition of effects of methylcholanthrene on mouse prostate in organ-culture by vitamin $A$ and its analogs. Cancer Res., 34: 1564-1571.
Lucy, J. A. 1969 Lysosomal membranes. In: Lysosomes in Biology and Pathology. J. T. Dingle and H. B. Fell, eds. North Holland, Amsterdam, pp. 313-341.

MacMahon, M. J., and G. H. Thomas 1973 Morphological changes of benign prostatic hyperplasia in culture. Brit J. Cancer, 27: 323-336.

Miller, H. C., and J. W. Alexander 1973 Protective effect of methylprednisolone against ischemic injury to the kidney. Transplantation, 16: 57-60.

Morfin, R. F., M. A. Alinpoulios, J. Chamberiain and P. Ofner 1970 Metabolism of testosterone-4-14C by the canine prostate and urinary bladder in vivo. Endocrinol., 87: $394-405$.

Pong, S., and L. Levine 1977 Prostaglandin biosynthesis and metabolism as measured by radioimmunoassay. In: The Prostaglandins. Vol. 3. P. W. Ramwell, ed. Plenum Press, N.Y. pp. 41-76.

Rundell, J. O., T. Sato, E. Wetzelberger, H. Eueda and D. Brandes 1974 Lysosomal enzyme release by vitamin A in 11210 leukemia cells. J. Natl. Cancer Inst., 52: 1237.1244.

Sinowatz, F., and C. J. Pierrepoint 1977 Hormonal effects on canine prostatic explants in organ culture. 3 . Endocrinol., 72: 53-58.

Starling, J. R., L. E. Rudolf, W. Ferguson and S. L. Wangensteel 1973 Benefits of methylprednisolone in the isolated perfused organ. Ann. Surg., 177:566-571.

Stephens, F. O., T. K. Hunt, E. Jawetz, M. Sonne and J. E. Dunphy 1973 Effect of cortisone and vitamin $A$ on wound infection. Am. J. Surg., 121: 569-571.

Sudhakaran, P. R., and P. A. Kurup 1974 Vitamin A and lysosomal stability in rat liver. J. Nutr., 104: 1466-1475.

Sweeny, P. R., and M. H. Hardy 1976 Ciliated and secretory epidermis produced from embryonic mammalian skin in organ culture by vitamin A. Anat. Rec., 185: 93-99.

Thomas, R. T., J. L. McCluskey, L. Potter and G. Weissman 1960 Comparison of the effects of papain and vitamin A on cartilage. I. The effects in rabbits. J. Exp. Med., 111: $705 \cdot 718$.

Weissmann, G. 1972 Lysosomal mechanisms of tissue injury in arthritis. Sem. Med. Beth. Israel Hosp., 286: 141-1.47.

Wenzel, D. G., and D. Acosta 1973 Permeability of lysosomes and mitochondria in cultured rat muscle and endothelial cells as affected by vitamin $A$, chlorpromazine, amphotericin B, and clofibrate. Res. Commun. Chem. Pathol. Pharmacol., 6: 689-700.

Wilson, J. W., and E. H. Leduc 1963 Mitochondrial changes in the liver of essential fatty acid-deficient mice. J. Cell Biol., 16: 281-296. 


\section{PLATE 1}

\section{EXPLANATION OF FIGURES}

4 Normal canine prostatic tissue before cultivation. All glandular acini are lined with a tall columnar epithelium. Stroma is sparse. $\times 385$.

5 An example of the variability seen in most explants after ten days of culture. Some glands still contain columnar epithelium (although vacuolated in comparison with the control, fig. 4) but low cuboidal and even squamous areas are common. $\times 385$.

6 An area of well maintained columnar epithelium in an explant from a dog pretreated with MP and cultivated for ten days in a medium containing $50 \mathrm{IU}$ retinol/ $\mathrm{ml}$. One third of all glands in this group exhibited this structural maintenance. $\times 385$.

7 A typical region of an explant from a dog not pretreated with MP nor given vitamin $A$ in culture. After ten days much of the glandular epithelium is in a regressed state. $\times 385$.

8 An area of an explant from a dog not pretreated with MP but cultivated for ten days in a medium containing $50 \mathrm{IU}$ vitamin $\mathrm{A} / \mathrm{ml}$. Much of the epithelium is well maintained, although regressed epithelium is also present. As is common in the absence of MP pretreatment, stromal cells are pyknotic or have completely disappeared by this time. Edema-like appearances are common in the stroma. $\times 385$. 

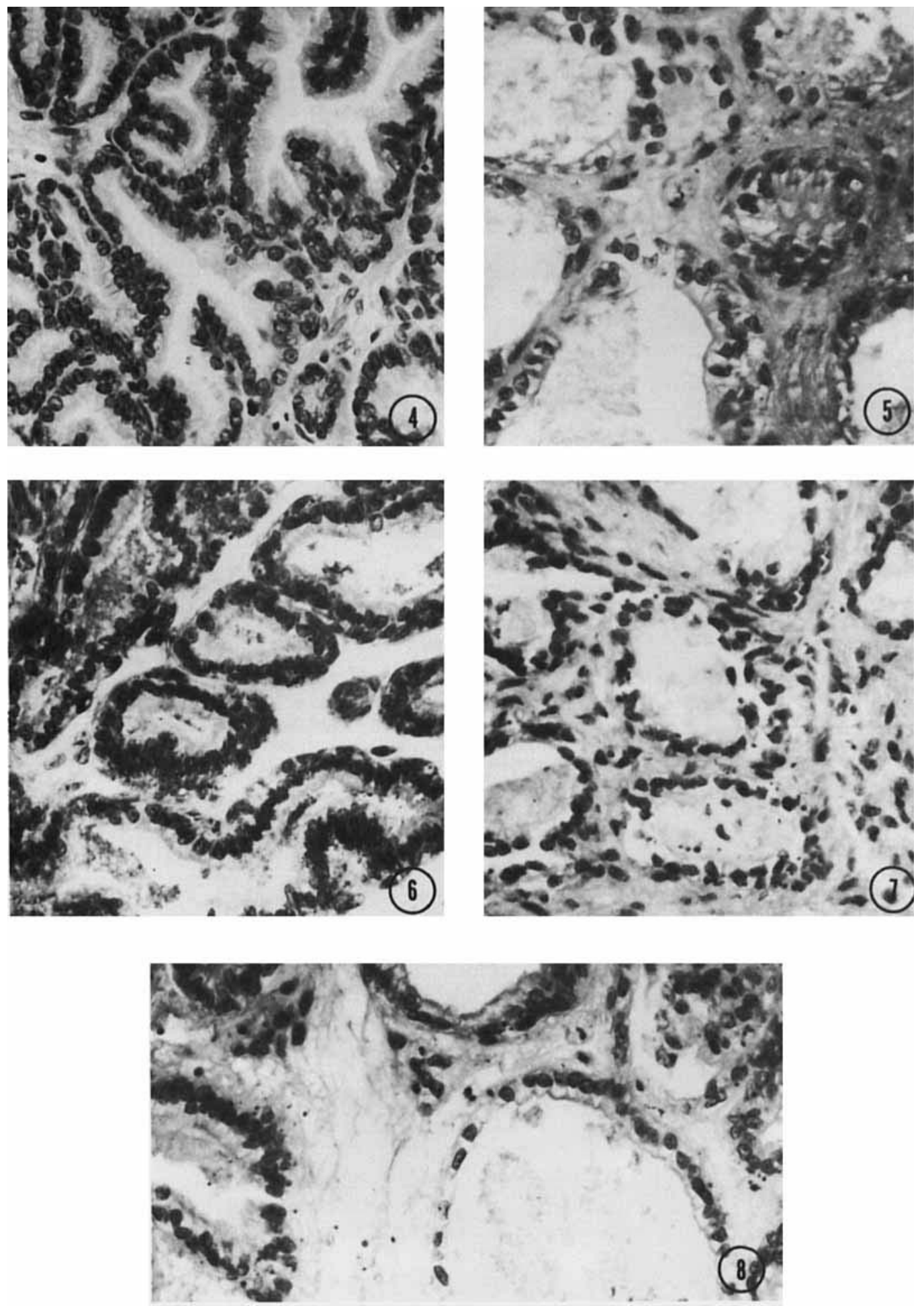